\title{
Alzheimer's disease dementia, amyloid imaging and underpinning fibrillar a? plaque associated pathology: are they aligned?
}

\begin{abstract}
We reported a case of 80-years-old man with Parkinsonism Disease Dementia (PDD) who experienced an improvement of hallucination with decreasing dose of madopar and non-pharmacological therapies. The patient was initially diagnosed as Parkinson's disease (PD) 15 years ago, and was medicated with madopar (dose of $250 \mathrm{mg}$ per day). The dose of madopar was gradually increased to $375 \mathrm{mg}$ per day during last 15 years because of decreasing curative effect. New symptoms of frequent visual hallucinations and aggressive behavior have emerged. After that, he was hospitalized to our department three months ago with diagnosis of PDD; we adjusted the dose of madopar to $250 \mathrm{mg}$ per day. Selegiline was later added to help managing the subsequent aggravation of his Parkinsonism. Quetiapine fumarate ( $25 \mathrm{mg}$ a day) was used to control his visual hallucinations and aggressive behavior. The frequency of visual hallucinations and attacking behaviours has been decreased gradually after drug treatment. We also prescribed the non-pharmacological therapies for the patient by means of musical therapy and cognitive psychotherapy. Two months later, the severity of his hallucination was alleviated. Furthermore, his NPI evaluating scores deceased from 42 to 12 , and MMSE evaluating scores increased from 15 to 22 . The pharmacological and non-pharmacological therapies may be good choices for PDD patient suffering from extrapyramidal symptoms and dementia.
\end{abstract}

Keywords: Amyloid $\beta$, Beta Amyloid, Dementia, Cerebral amyloid angiopathy, Mild Cognitive Impairment, Cerebral Amyloid Angiopathy, Frontotemporal Dementia, Positron Emission Tomography
Volume 2 Issue 2 - 2015

\author{
Igor D Grachev, ${ }^{1,2}$ \\ 'Clinical Development and Medical Affairs Consultant, Novartis \\ Consumer Health, USA \\ ${ }^{2}$ Clinical and Medical Consultant, Genpact Pharmalink, USA
}

\begin{abstract}
Correspondence: Igor D Grachev, MD, PhD., Novartis Consumer Health, Parsippany, New Jersey, USA, Genpact Pharmalink, Short Hills, New Jersey, USA,
\end{abstract}

Email grachevi@hotmail.com

Received: September 10, 2014 | Published: April 09, 2015
Abbreviations: AD, Alzheimer's Disease; CAA, Cerebral Amyloid Angiopathy; FTD, Frontotemporal Dementia; aMCI, amnestic Mild Cognitive Impairment; CERAD, Consortium to Establish a Registry for Alzheimer's Disease; NIA, National Institute on Aging; A $\beta$, Amyloid $\beta$; APP, A $\beta$ Precursor Protein; TL, Temporal Lobe; IPL, Inferior Parietal Lobule; CDR, Clinical Dementia Rating; IHC, Immunohistochemistry

\section{Introduction}

The goal of this review is to summarize the literature about Alzheimer's disease (AD) diagnosis at the level of clinical, histopathological examinations and amyloid PET imaging, which is important not only to clinicians, but also to scientists who develop and test new therapies and biomarkers for AD. Dementia is a clinical syndrome characterized by multiple cognitive deficits, including memory impairment and deficits in speech, motor ability, and ability to recognize or identify objects. Alzheimer's disease (AD) is the most common form of dementia and is characterized by the presence of fibrillar amyloid $\beta(\mathrm{A} \beta)$ in the form of extracellular plaques and hyperphosphorylated tau protein in the form of intracellular neurofibrillary tangles in specific brain regions. The definitive diagnosis of $\mathrm{AD}$ requires neuropathological examination of brain tissue. $^{1}$

\section{Fibrillar A $\beta$ plaque associated pathology}

Dementia syndromes can be categorized into those associated with fibrillar $A \beta$ plaques and those not associated with fibrillar $A \beta$ plaques. Alzheimer's disease is the prototypic dementia associated with fibrillar $\mathrm{A} \beta$ plaques and their presence with neurofibrillary tangles is necessary for a confirmatory histopathological diagnosis. ${ }^{2}$ Other, $A \beta$-associated syndromes are cerebral amyloid angiopathy $(\mathrm{CAA})^{3}$ and Dementia with Lewy bodies (DLB). ${ }^{4}$ Dementia syndromes not associated with A $\beta$ deposition include Frontotemporal Dementia (FTD) and dementia associated with Parkinson's disease., ${ }^{5,6}$ Amyloid deposition may be present in many cognitively normal elderly people ${ }^{7,8}$ and more frequently in people with evidence of the $\mathrm{AD}$ precursor syndrome amnestic Mild Cognitive Impairment (aMCI). ${ }^{9}$

\section{Diagnostic criteria for Alzheimer's disease}

There are several published histopathologic criteria for the diagnosis of AD. These criteria are necessary to distinguish between pathologic changes associated with healthy aging versus those indicative of AD. ${ }^{1}$ The most commonly used criteria in clinical pathology practice and research are those developed by the Consortium to Establish a Registry for Alzheimer's Disease (CERAD) ${ }^{10}$ and by the National Institute on Aging (NIA)-Reagan criteria (National Institute on Aging and Reagan Institute). ${ }^{11}$ These criteria establish thresholds for the likelihood of an $\mathrm{AD}$ diagnosis. Many experts using the CERAD scoring criteria, which have been employed by Ikonomovic in his published work validating the amyloid detection properties of the PET tracer $\left[{ }^{[\sup >11</ \sup >\mathrm{C}}\right] \mathrm{PiB} .{ }^{12}$

\section{$A \beta$ and the pathophysiology and neuropathology of Alzheimer's disease}

Definitive diagnosis of $\mathrm{AD}$ relies on the demonstration of amyloid plaques and neurofibrillary tangles at autopsy. ${ }^{10}$ Amyloid plaques are composed of the 40-42 amino acid long A $\beta$ peptides. ${ }^{13}$ Neurofibrillary tangles are mainly composed of a hyperphosphorylated form of the microtubule-associated protein, tau. ${ }^{14} \mathrm{~A} \beta$ also can be found deposited around small arterioles in the form of cerebrovascular amyloid. ${ }^{15}$ Plaques occur earliest in the neocortex where they are relatively 
evenly distributed. ${ }^{16}$ Tangles appear first in limbic areas such as the transentorhinal cortex and progress in a predictable topographic pattern to the neocortex. ${ }^{17}$

A growing consensus points to deposition of $A \beta$ plaques as a central event in the pathogenesis of AD. The single, most important piece of evidence for this "amyloid cascade hypothesis" of AD is the demonstration that several mutations in the $\mathrm{A} \beta$ precursor protein (APP) gene on chromosome 21 are associated with early onset AD. ${ }^{18}$ While a very small number of families are affected with this form of $\mathrm{AD}$, the disease is phenotypically very similar to the more common sporadic form of $\mathrm{AD}$, save only its early age of onset. ${ }^{19}$ Further genetic support for the amyloid cascade hypothesis comes from the finding that the most common form of autosomal dominant $\mathrm{AD}$ is associated with mutations in the presenilin-1 gene on chromosome 14 which codes for a protein strongly implicated to be an essential component of the "gamma-secretase" enzyme complex responsible for C-terminal cleavage of $\mathrm{A} \beta$ from its precursor, APP. ${ }^{20} \mathrm{In}$ contrast, no mutation in the tau protein has been shown to cause AD. Mutations in tau (chromosome 17) are linked to frontotemporal dementia with Parkinsonism. ${ }^{14}$

A sizable body of work existed on the regional distribution of $\mathrm{A} \beta$ plaques in post-mortem tissue prior to the first attempts to image amyloid in vivo. Several investigators including Mann (1985), Arnold et $\mathrm{al}^{.1}$ and Braak and Braak $^{22}$ performed extensive post-mortem studies to define the temporal progression and regional distribution of $\mathrm{A} \beta$ plaque deposition in $\mathrm{AD}$. Arnold et al. ${ }^{21}$ mapped the distribution of neurofibrillary tangles and amyloid plaques surrounded by dystrophic neuritis in the brains of patients with AD. Compared to neurofibrillary tangles, neuritic plaques were more evenly distributed throughout the cortex with fewer in limbic periallocortex and allocortex: the areas with greatest neurofibrillary tangle density. The cerebellum is notably free of neuritic plaques in $\mathrm{AD}$, although diffuse amyloid deposits that do not label with fibrillar dyes such as Congo red or Thioflavin-S are commonly observed in the cerebellum..$^{23,24}$

Braak and colleagues are well known for their neurofibrillary tangle staging system, and they have also done extensive work on plaque pathology. ${ }^{16,22,25}$ Their work can be separated into:

a. A study of $A \beta$ deposition across the entire brain, midbrain, pons and medulla. ${ }^{16}$

\section{b. A study of the stages of $A \beta$ deposition across the neocortex. ${ }^{22}$}

c. A detailed study of the stages of $A \beta$ deposition in the temporal lobe. ${ }^{25}$

Thal et al. ${ }^{16}$ described five "phases" of amyloid plaque deposition across the entire brain. Of course, these post-mortem studies are cross-sectional and the temporal pattern of deposition is inferred from patterns of deposition observed in brains with different levels of involvement. For example, if $A \beta$ deposition in region- $X$ is always present when there is deposition in region-Y, but $A \beta$ deposition in region- $X$ can be found in the absence of plaques region- $Y$, then it is presumed that deposition in region-X temporally precedes that in region-Y.

Phase- 1 is the neocortical phase in which $A \beta$ deposits are found exclusively in the neocortex. Although plaques can be in the frontal, parietal, temporal or occipital neocortex in Phase-1, an earlier study by Braak and Braak ${ }^{22}$ suggested plaques typically appear first in the basal neocortex, most frequently in poorly myelinated areas of the anterior temporal and inferior frontal cortex. ${ }^{22}$ They referred to this as "Stage-A". Thal et al. ${ }^{16}$ also detailed the sequence of $A \beta$ deposits in the temporal lobe (TL). ${ }^{25}$ In Phase-1, plaques are confined to the basal, lateral temporal neocortex (TL-1 stage).

In Phase-2, $A \beta$ deposits can be found in allocortical brain regions including entorhinal cortex, CA1 and the insular cortex (corresponding to the TL-2 stage). Plaques are sometimes seen in the amygdala, the cingulate gyrus, the presubicular region, the molecular layer of the fascia dentata, and small patches of subpial band-like amyloid appear in the frontal, parietal, temporal, and occipital neocortex (overlapping with the TL-3 stage). All other areas do not show A $\beta$ in this phase.

In Phase- $3, A \beta$ plaques are seen in subcortical regions including the caudate, putamen, claustrum, basal forebrain nuclei, substantia innominata, thalamus, hypothalamus, lateral habenular nucleus and white matter just beneath the cortex. In the TL-3 stage, A $\beta$ deposits are found in the molecular layer of the fascia dentata, and bandlike A $\beta$-deposits occur in the subpial portion of the molecular layer of both the entorhinal region and the temporal neocortex. In addition, confluent $A \beta$-deposits appear in the parvopyramidal layer of the presubicular region.

In Phase- $4, A \beta$ deposits can be found in the inferior olivary nucleus, the reticular formation of the medulla oblongata, the substantia nigra, CA4, the central gray of the midbrain, the colliculi superiores and inferiores and the red nucleus. However, within the inferior olivary nucleus, the reticular formation of the pons and the medulla oblongata, and the red nucleus there are often only one to three plaques in the entire anatomic structure. The TL- 4 stage parallels Phase- 4 and plaques can be found in CA4 in addition to the other temporal areas listed above.

In Phase-5, $A \beta$ deposits can be found in the reticular formation of the pons, the pontine nuclei, the central and dorsal raphe nuclei, the locus coeruleus, the parabrachial nuclei, the dorsal tegmental nucleus, the reticulotegmental nucleus of the pons, and the cerebellum. In the cerebellum, $A \beta$ most frequently occurs in the molecular layer and $A \beta$ deposits were never found in the dentate nucleus or other cerebellar nuclei. These cerebellar deposits are rarely fibrillar and accordingly do not stain with fibrillar dyes as previously mentioned.

Progression of neocortical amyloid deposits from Stage-A to Stage-B appears to occur in parallel to Phases-2 and -3. Further progression in the neocortex to Stage-C parallels Phases 3-5. It should be emphasized that the post-mortem models of amyloid deposition discussed above were completed before and therefore were not influenced by any in vivo amyloid imaging studies. The patterns described form a basis against which to judge the performance of amyloid imaging tracers. However, several important points that stem from this extensive body of neuropathological data often go unrecognized. First, $A \beta$ deposition starts in the neocortex, not in the medial portions of the temporal lobe. Second, the hippocampus contains smaller amounts of fibrillar $\mathrm{A} \beta$ deposits than the surrounding cortical areas, even at later stages. Third, striatal $\mathrm{A} \beta$ deposition occurs in $80 \%$ of Phase- 3 cases and $100 \%$ of Phase- 4 and Phase- 5 cases. ${ }^{16}$ Since all of the AD cases included in the Thal et al. ${ }^{16}$ study were in Phases 3-5, this means nearly all their AD cases had striatal amyloid deposition. Extensive amyloid deposition has been reported to occur in the striatum of virtually all AD patients in several prior studies as well. ${ }^{17,26-28}$ Striatal plaque deposition appears to occur early in the progression of $\mathrm{AD}$ pathology and coincides with neocortical pathology and cognitive changes..$^{28}$ While neuritic elements have been described in ventral striatum, ${ }^{26}$ most striatal $A \beta$ deposits are not associated with dystrophic neuritis. ${ }^{26,27}$ Despite this poorly understood absence of neuritic changes in the striatum, striatal plaques appear to be fibrillar as evidenced by the fact that they are stained well by 
fibril-specific dyes such as the Congo red derivative, $\mathrm{X}-34^{29}$ and the Thioflavin-T derivative, 6 -CN-BTA- $1 .^{30}$

Braak and colleagues proposed a sequential deposition of $A \beta$, but did not quantify the $A \beta$ load in the various brain areas. Quantification is done biochemically with enzyme-linked immunosorbent assay (ELISA). Naslund et al..$^{31}$ have used A $\beta$ ELISA analyses to show that frontal cortex contains significantly more $\mathrm{A} \beta$ than either the temporal or parietal cortex at Clinical Dementia Rating (CDR) stages 0.5-2 (2000). Thus, the frontal cortex is one of the earliest sites of brain $A \beta$ deposition and typically has the highest concentration of insoluble $\mathrm{A} \beta$ deposits of any brain area. The most medial portions of the temporal lobe, such as the hippocampus, typically show lower levels of $A \beta$ deposition and these occur later than those observed in the neocortex. ${ }^{22}$

\section{Neuropathological studies of aging}

Amyloid deposition is associated with aging and in autopsy studies, a substantial proportion of cognitively normal elderly individuals have been found to have both amyloid deposition and evidence of pathological features of AD. ${ }^{32}$ Neuropathological studies have demonstrated cortical $\mathrm{Ab}$ plaques decades before the usual onset of dementia that almost invariably develops in these individuals, ${ }^{33}$ suggesting that neuropathological changes precede the clinical expression of AD by at least a decade. ${ }^{34,35}$ As a result, Jack et al ${ }^{36}$ have recently proposed that $\mathrm{AD}$ can be considered a biphasic disease: the first phase characterized by the development of fibrillar $A \beta$ plaques which is then followed by a phase of neurodegeneration. Amyloid is not often found in individuals less than 55 years old, except in Down's syndrome and some other genetic syndromes associated with AD.

\section{Relationship of Alzheimer's Disease Clinical Diagnosis to Pathology}

Even in expert centers, concordance between clinical diagnosis of $\mathrm{AD}$ and pathological findings is not exact. Previous clinicalpostmortem correlation studies, have reported that $\sim 10-15 \%$ of $\mathrm{AD}$ subjects diagnosed in expert centers show no signs of $A \beta$ pathology. ${ }^{37}$ The diagnostic precision of community physicians is lower when rigorously assessed and compared to autopsy. For example, in a community-based brain bank program, only $77 \%$ of 234 clinically diagnosed $\mathrm{AD}$ subjects received a pathological diagnosis of $\mathrm{AD}$ and $52 \%$ of those diagnosed with a non-AD dementia actually had pathological AD. ${ }^{38}$

It is critical to choose a pathological assessment approach that can accurately and quantitatively assess the amount of amyloid in the brain (Figure 1). For continuous comparisons with in vivo amyloid imaging probes that result in the determination of a Pearson's or Spearman's correlation coefficient, a continuous measure of brain A $\beta$ load is needed. The most accurate and least biased approach for this purpose is the assessment of total $\mathrm{A} \beta$ load by an antibody specific to $\mathrm{A} \beta$ such as the widely employed 4G8 antibody directed at the midportion (residues 17-24) of the $A \beta$ peptide. This approach will have little bias because it will detect almost all forms of deposited $A \beta$ and has been shown to be as sensitive as the Bielschowsky technique at assessing neuritic plaque load ${ }^{39}$ and will also detect non-neuritic plaques (i.e., diffuse, amorphous and fleecy $\mathrm{A} \beta$ deposits) and CAA as well. A recent consensus panel of the BrainNet Europe consortium chose 4G8 as the optimal antibody for all plaques including diffuse/ fleecy plaques. ${ }^{40}$ The latter fact is likely to result in some discrepancies between an in vivo measure of fibrillar $\mathrm{A} \beta$ and a postmortem measure of total $A \beta$ because the $4 \mathrm{G} 8$ antibody will detect more $A \beta$ deposits than are detectable by any measure specific for fibrillar $A \beta$.

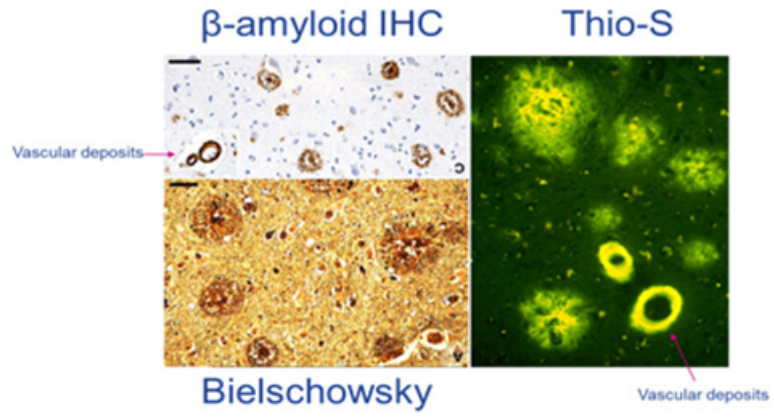

Figure I Alzheimer's disease histochemistry and immunohistochemistry.

Many investigators have reported methods for defining an abnormal or amyloid-positive PET scan. For $\left[{ }^{18} \mathrm{~F}\right]$ flutemetamol or other amyloid probed, this can be done visually and/or from the quantitative SUVR values. It is important to note that the " $A \beta$-positive cutoff" is meant to distinguish between $A \beta$-positive and $A \beta$-negative subjects regardless of clinical diagnosis as opposed to what might be called an " $\mathrm{AD}$ cutoff" which is meant to distinguish between clinical AD and clinical normality.

\section{Histopathology (immunohistochemistry) threshold}

In order to dichotomize a postmortem measure of $A \beta$ load to determine a pathology threshold, many experts rely on the more representative measure of $\mathrm{A} \beta \mathrm{IHC}$ with the $4 \mathrm{G} 8$ antibody. The use of this antibody has been shown to achieve a high level of concordance for assessment of the presence or absence of plaques and CAA on 2 $\mathrm{mm}$ diameter tissue core sections in microarrays in a large multicenter study. ${ }^{40}$ This shows that good results can be achieved from very small tissue samples such as those obtained at brain biopsy. Alafuzoff et al. ${ }^{40}$ defined the "presence" of plaques as $\sim 10$ plaques per 100x field. They also defined higher categories of "moderate" ( $\sim 40$ plaques per $100 \mathrm{x}$ field) and "many" ( $\sim 100$ plaques per $100 x$ field). These categories should not be equated to the CERAD sparse, moderate and frequent mainly because the IHC method detects all forms of deposited $\mathrm{A} \beta$, not just neuritic plaques. Thus, Alafuzoff et al. ${ }^{40}$ defined a threshold for the "presence" of plaques at a level $\sim 1 / 10^{\text {th }}$ the maximum plaque load. Although there was some disagreement among 21 raters in the Alafuzoff et al. ${ }^{40}$ study as to whether there were some-or-moderate and moderate-or-many plaques in a tissue sample, there was complete agreement as to whether there was a "presence" or "absence" of plaques using these definitions. ${ }^{40}$ This presence-absence distinction is most relevant for clinical research and this appears to be a good technique with which to generate a sensitive dichotomous postmortem measure of the presence of significant $A \beta$ deposition which can be used for comparison to the dichotomized $\left[{ }^{18} \mathrm{~F}\right]$ flutemetamol or any other amyloid probe result (visual read or quantitative). Unfortunately, the Alafuzoff et al. ${ }^{40}$ study did not define the cutoff for "presence" of plaques by percent area. However, one can reasonably extrapolate the Alafuzoff et al. ${ }^{40}$ results to percent area by two methods.

First, one can compare the plaque count necessary for the Alafuzoff et al. ${ }^{40}$ definition of "many" $(\sim 100)$ to the upper limit of percent $A \beta$ plaques area observed in $\mathrm{AD}$ brains. Ikonomovic et al. ${ }^{12}$ have found percent area of $\mathrm{A} \beta$ of up to $\sim 15 \%$ in a typical $\mathrm{AD}$ patient ${ }^{12}$ and others have reported up to $27.1 \pm 11.0 \%$ area in $\mathrm{AD}$ patients and $14.5 \pm 6.0 \%$ area in non-demented elderly controls with "high amyloid plaque density". ${ }^{41}$ Using a cutoff similar to Alafuzoff et al. ${ }^{40}$ at approximately $1 / 10^{\text {th }}$ the maximum load would yield a cutoff of $1.5-3 \%$ plaque area 
to define the "presence" of significant $\mathrm{A} \beta$ load for the purposes of a dichotomization of the presence/absence of $A \beta$ deposition by IHC. Second, one could estimate the percent area occupied by 10 average $\mathrm{A} \beta$ plaques in a $100 \mathrm{x}$ microscopic field. A typical 100x field of view covers $\sim 2.5 \mathrm{~mm}^{2}$. An average A $\beta$ plaque is $50-100 \mu \mathrm{m}$ in diameter or $0.002-0.008 \mathrm{~mm}^{2}$ in area. This would equate to $\sim 1-3 \%$ plaque area. Both of these extrapolation methods give similar results and a conservative cutoff can be set at $1.5 \%$ plaque area.

It is well recognized that postmortem immunohistochemical techniques using high-affinity monoclonal antibodies will, by their nature, always be more sensitive than any in vivo amyloid imaging technology. Thus, it is recognized that some level of amyloid deposition- detectable at autopsy by these sensitive in vitro techniqueswill not be detectable in vivo.

It is well recognized that postmortem immunohistochemical techniques using high-affinity monoclonal antibodies will, by their nature, always be more sensitive than any in vivo amyloid imaging technology. Thus, it is recognized that some level of amyloid deposition- detectable at autopsy by these sensitive in vitro techniqueswill not be detectable in vivo.

It is well recognized that postmortem immunohistochemical techniques using high-affinity monoclonal antibodies will, by their nature, always be more sensitive than any in vivo amyloid imaging technology. Thus, it is recognized that some level of amyloid deposition- detectable at autopsy by these sensitive in vitro techniqueswill not be detectable in vivo.

\section{Histopathology versus in vivo amyloid PET imaging}

There are three histopathological criteria that have been widely used in the diagnosis of $\mathrm{AD}$. While it might seem that one of these criteria might be a good correlate for in vivo fibrillar $\mathrm{A} \beta$ imaging, each has serious weaknesses relative to IHC that make them inappropriate for a correlate with in vivo A $\beta$ PET imaging.

The Khachaturian criteria $^{42}$ has some relationship to in vivo assessment of fibrillar $\mathrm{A} \beta$ pathology since a diagnosis of $\mathrm{AD}$ by these criteria relies solely on assessment of $A \beta$ plaque numbers in multiple brain areas (i.e., neurofibrillary tangle counts that are not measured by fibrillar $\mathrm{A} \beta$ imaging agents are not considered in the assessment). However, the cutoff for a diagnosis of AD varies according to age (higher plaque counts are required with increasing age) and by the presence or absence of a clinical diagnosis of dementia (fewer plaques are necessary in the presence of a clinical diagnosis). Clearly, an in vivo measure of fibrillar $A \beta$ pathology must produce the same quantitative result in the brains of any two subjects who have identical plaque loads regardless of the age or clinical history of the two subjects. The Khachaturian criteria have largely been supplanted in the US by one of the two criteria discussed below.

Derived from the Khachaturian criteria, the CERAD criteria ${ }^{10}$ also have some appeal as a postmortem comparator to in vivo assessment of fibrillar $\mathrm{A} \beta$ since these criteria also rely solely on plaque counts. CERAD also has adjustments for age and clinical diagnosis, but these criteria have several other serious shortcomings for use as a comparator for in vivo fibrillar $A \beta$ imaging. First, plaques are only semi-quantified into four categories: none, sparse, moderate or frequent. This does not provide a good continuous measure for comparison to a continuous measure such as that obtained from a PET measure of fibrillar A $\beta$ burden. Second, plaques are only quantified in three cortical regions: the middle frontal gyrus, the inferior parietal lobule (IPL), and the superior and middle temporal gyri and the total area sampled in these three regions is relatively small since this is done on a microscopic scale. The microscopic field (typically only several $\mathrm{mm}^{2}$ in area) with the highest plaque frequency gives the score to each of the three areas and the final CERAD score is derived from the highest score in any of these three regions. Thus, the CERAD score can be driven by a single microscopic field that may not be representative of the brain as a whole. That is, a section of tissue that is several $\mathrm{mm}^{2}$ in area and only microns thick, representing less than $0.01 \%$ of the total neocortical volume can drive the CERAD score. This obviously opens the possibility of serious sampling bias problems. Third, CERAD plaque counts are exclusively from neuritic plaques only and are typically identified with a silver stain such as that of Bielschowsky. ${ }^{10,43,44}$ Neuritic plaques are $\mathrm{A} \beta$ plaques surrounded by dystrophic neuritic processes containing tau proteins. ${ }^{45}$ Although the Bielschowsky stain can detect all forms of plaques, its use here is "tuned" to the detection of neuritic plaques and the raters are trained to count only the neuritic plaques. Braak et al ${ }^{46}$ have stated that "a considerable proportion of the amyloid plaques do not correspond to and should carefully be distinguished from 'primitive', 'mature', and 'burned out' types of neuritic (senile) plaques" ${ }^{46}$ Non-neuritic plaques, even those appearing diffuse, can contain substantial fibrillar amyloid. ${ }^{47}$ To quote the late neuropathologist, H. M. Wisniewski, CERAD scores reflect "a single microscopic field of maximal involvement, rather than on more generalized measures of regional neuropathology, and refer exclusively to neuritic rather than $\beta$-amyloid plaques". ${ }^{48}$ Thus, the final CERAD rating is likely to be a poor representation of the total fibrillar $A \beta$ load of a brain and would thus be a poor comparator for an in vivo technique that was intended to be a quantitative measure of brain fibrillar $A \beta$.

The NIA/Reagan criteria (1997) differ most from the previous two sets of criteria by the inclusion of neurofibrillary tangle assessment in the determination of the likelihood that $\mathrm{AD}$ is present (Table 1). That is, the Braak and Braak staging system for neurofibrillary tangles plays a role in the diagnosis. ${ }^{17}$ Assessment of plaques is made in exactly the same way as CERAD. Thus, the NIA/Reagan criterion essentially takes the CERAD criteria and appends a Braak tangle stage to provide a purely pathological diagnosis and an estimate about the probability that $\mathrm{AD}$ is present. The original NIA/Reagan criteria described three levels of likelihood: low (Braak I-II and sparse plaques), intermediate (Braak II-IV and moderate plaques) and high (Braak V-VI and frequent plaques). It is apparent that these three combinations represent only 3 of the 9 possible combinations of the 3 Braak stages with the 3 CERAD plaque frequencies. Others have proposed $3 \times 3$ tables to address all 9 possibilities. ${ }^{48}$

Table I NIA-Reagan and CERAD criteria for Alzheimer's disease diagnosis

\begin{tabular}{llll}
\hline NIA-reagan criteria & \multicolumn{2}{l}{} \\
& CERAD neuritic plaque score & \\
\hline Braak \& Braak stage & Sparse & Moderate & Frequent \\
I-II & Low & Low-Intermediate & High \\
III-IV & Low-Intermediate & Intermediate & High \\
V-VI & Intermediate & High & High \\
\hline
\end{tabular}

*Note: Braak criteria include only neuropathology (neurofibrillary tangles [NFT], and does not account for A $\beta$ plaques). CERAD criteria include both plaque neuropathology and clinical criteria (accounts for highest neuritic plaque count). NIA Reagan criteria include both CERAD and Braak neuropathology (accounts for both but didn't correlation with the $A \beta$ plaque load).

Although the NIA/Reagan criteria is well-suited for the neuropathological diagnosis of $\mathrm{AD}$ and has been used frequently and effectively for this purpose, ${ }^{49}$ it appears to be the worst of the three criteria for the purpose of a comparator to a quantitative in 
vivo measure of fibrillar $A \beta$ pathology. This is because it retains all of the semi-quantitative and sampling bias disadvantages of the CERAD criteria described above, and adds the additional disadvantage of mixing neurofibrillary tangle pathology into the final likelihood score. Thus, using the Table 1, a two brains could have exactly the same load of $\mathrm{A} \beta$ pathology (e.g., resulting in a moderate CERAD plaque score) yet one brain could get a "low likelihood" and another get a "high likelihood" and this difference would be driven solely by the neurofibrillary tangle pathology that has nothing at all to do with the accuracy of the in vivo PET tracer aimed at fibrillar A $\beta$ pathology.

It also is important to note that another important component of brain $A \beta$ load is completely ignored in all three of these diagnostic criteria, cerebrovascular amyloid or cerebral amyloid angiopathy (CAA). This important lesion is present in over two-thirds of patients with AD. ${ }^{50}$ Amyloid imaging tracers have been shown to label CAA well in postmortem tissue ${ }^{12,51}$ and binding to CAA likely contributes to the in vivo signal. ${ }^{52}$ The lack of representation of this form of $\mathrm{A} \beta$ deposition in any of the above mentioned diagnostic criteria underscores the inappropriateness of these criteria as a comparator for a quantitative in vivo measure of fibrillar $A \beta$ load. Proposed classification criteria for AD dementia from the National Institute on Aging and the Alzheimer's Association workgroup ${ }^{53}$ propose the following terminology for classifying individuals with dementia caused by AD: (a) Probable AD dementia, (b) Possible AD dementia, and (c) Probable or possible AD dementia with evidence of the AD pathophysiological process. This is more advanced classification coupled with a more comprehensive approach utilizing knowledge of biomarkers (including amyloid PET imaging and CSF) to the AD dementia diagnostic process.

However, the important question is how well does $\left[{ }^{18} \mathrm{~F}\right]$ flutemetamol or $\left[{ }^{<\sup >11</ \text { sup }>C}\right] \mathrm{PiB}$ or any other PET diagnostic probe retention in the brain (expressed as SUVR) represent the amount of amyloid deposition in the brain (Figure 2). Previous studies of $\left[{ }^{18} \mathrm{~F}\right]$ flutemetamol in living subjects with normal pressure hydrocephalus (NPH) needing ventriculo-peritoneal shunting or intracranial pressure monitoring showed a good correlation between imaging via standard uptake value ratio (SUVR) and neuritic plaque burden assessed with Bielschowsky silver stain (Spearman's $\mathrm{r}=0.61-074, \mathrm{p}=0.0001$ ). Overall sensitivity and specificity of the test by majority read were 93 and $100 \% \cdot{ }^{54-57}$ Cortical uptake of $\left[{ }^{18} \mathrm{~F}\right]$ flutemetamol was assessed visually by blinded reviewers, and also quantitatively via SUVR in specific neocortical regions in relation to either cerebellum or pons reference region. $A \beta$ pathology in the biopsy specimen (standard of truth (SoT)) was measured using Bielschowsky silver and thioflavin $\mathrm{S}$ plaque scores, percentage area of grey matter positive for monoclonal antibody to $\mathrm{A} \beta$ (4G8), and overall pathology impression. Authors set out to find which pair(s) of PET SUVR and pathology SoT endpoints matched best, whether quantitative measures of $\left[{ }^{18} \mathrm{~F}\right]$ flutemetamol PET were better for predicting the pathology outcome than blinded image examination (BIE), and whether there was a better match between PET image findings in retrospective vs. prospective studies. Of the 24 possible endpoint/SoT combinations, the one with composite-cerebellum SUVR and SoT based on overall pathology had the highest Youden index (1.000), receiver operating characteristic area under the curve (1.000), sensitivity (1.000), specificity (1.000), and sum of sensitivity and specificity for the pooled data as well as for the retrospective and prospective studies separately (2.00, for all 3). The BIE sum of sensitivity and specificity, comparable to that for quantitation, was highest using Bielschowsky silver as SoT for all SUVRs (ipsilateral, contralateral, and composite, for both reference regions). The composite SUVR had a $100 \%$ positive predictive value (both reference regions) for the overall pathology diagnosis. All
SUVRs had a $100 \%$ negative predictive value for the Bielschowsky silver result. Bielschowsky silver stain and overall pathology judgment showed the strongest associations with imaging results. ${ }^{58}$

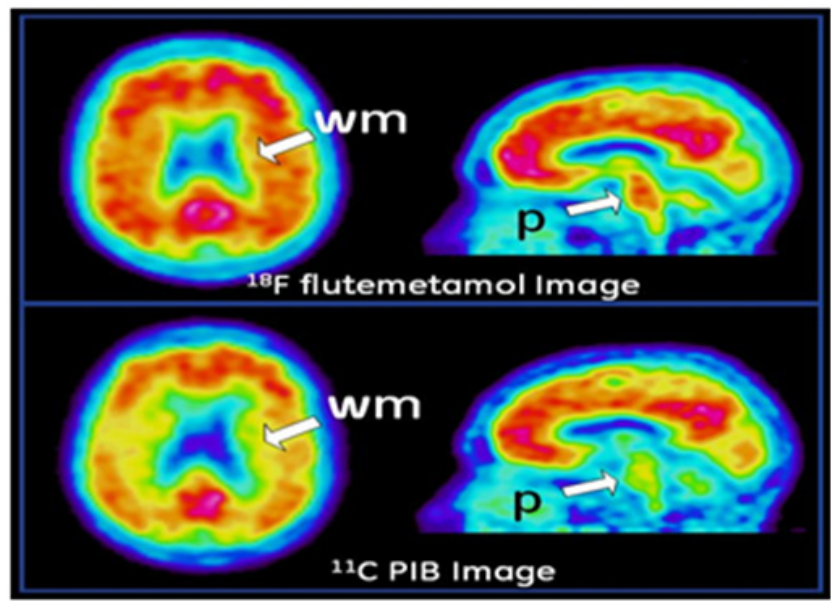

Figure $2\left[{ }^{18} \mathrm{~F}\right]$ flutemetamol (upper row) and ["IC]PiB (lower row) images of the same AD patient. The images show similar enhanced signal in the grey matter cortical regions of the brain due to b-amyloid plaque loading, with the main differences being that the $\left[{ }^{18} \mathrm{~F}\right]$ flutemetamol has a higher signal in the white matter ( $w m)$ and the pons $(p)$ than the $[" C]$ PiB images.

Clark et al. ${ }^{59}$ evaluated associations between PET imaging of amyloid plaques and amyloid- $\beta$ pathology measured at autopsy in 59 terminally ill subjects who had a life expectancy of less than 6 months. The sensitivity and specificity of florbetapir (Amyvid, Lilly USA, LLC, Indianapolis, IN) PET imaging for detection of moderate to frequent plaques were $92 \%$ and $100 \%$, respectively, in people who had autopsy within 2 years of PET imaging, and $96 \%$ and $100 \%$, respectively, for those who had autopsy within 1 year. Amyloid assessed semiquantitatively with florbetapir PET was correlated with the post-mortem amyloid burden in the participants who had an autopsy within 2 years (Spearman $\rho=0.76 ; \mathrm{p}<0.0001)$ and within 12 months between imaging and autopsy $(0.79 ; p<0.0001)$. Curtis et al. ${ }^{60}$ determined the sensitivity and specificity of PET imaging with flutemetamol (Vizamyl; GE Healthcare, Princeton, NJ) in terminally ill patients with a life expectancy of less than 1 year. Images were classified as positive or negative for $\beta$-amyloid by 5 readers who were blind to patient information. From 176 patients who had evaluable images, 68 patients (38\%) died during the study, were autopsied, and had neuritic plaque levels determined. Sensitivity without computed tomography was $81 \%$ to $93 \%$ (median, $88 \%$ ). Median specificity was $88 \%$, with 4 of 5 of the readers having specificity greater than $80 \%$. These results were similar to observed above for $\left[{ }^{18} \mathrm{~F}\right]$ florbetapir in terminally ill patients subjects with a life expectancy of less than 6 months [59], and were similar to results observed in NPH subjects when cortical tissues were taken during neurosurgery and analyzed using the same tracer $\left[{ }^{18} \mathrm{~F}\right]$ flutemetamol. ${ }^{54-57}$

In study by Yotter et al. ${ }^{61}$ authors examined the spatial patterns of amyloid deposition throughout the brain using Pittsburgh Compound Blue $(<$ sup $>11</$ sup $>$ C-PiB $)$ PET data from the Baltimore Longitudinal Study of Aging. Results indicate that the spatial pattern of amyloid deposition is related to cognitive performance and may be more informative than a biomarker reflecting total amyloid burden, the use of which is the current practice. This finding has broad implications for our understanding of the relationship between cognitive decline/resilience and amyloid deposition, as well as for the use of amyloid imaging as a biomarker in research and clinical applications. 
In two double-blind, randomized (Bapineuzumab or placebo), phase 3 trials involving patients with mild-to-moderate Alzheimer's disease--one involving carriers of the apolipoprotein E (APOE) $\varepsilon 4$ allele and the other involving noncarriers, ${ }^{62}$ between-group differences were observed with respect to $<$ sup $>11<$ sup $>$ C-PIBPET and cerebrospinal fluid phospho-tau concentrations in APOE $\varepsilon 4$ allele carriers but not in noncarriers. Interestingly enough, there were no significant between-group differences in the primary outcomes--change from baseline in the ADAS- $\operatorname{cog} 11$ and DAD scores (bapineuzumab group minus placebo group), suggesting that using amyloid imaging probes can be reasonable to improve sensitivity of outcome measures in testing future disease-modifying therapies. It would require pharmaceutical and diagnostic/health care companies, and academic centers to validate amyloid imaging probes to be used as a regulatory-accepted endpoints.

Amyloid imaging probes performance has been discussed from available clinical trials assessing diagnostic sensitivity, specificity, and accuracy; this type of technology recognized as a sensitive outcome measure in testing future therapies. The future directions in which field should move forward would require to improve the clinical diagnosis of $\mathrm{AD}$ and to validate amyloid imaging probes to be used as regulatory-accepted endpoints in disease-modifying trials. These efforts are ongoing.

\section{Acknowledgments}

None.

\section{Conflicts of interest}

None.

\section{References}

1. Arnaldi D, Morbelli S, Morrone E, et al. Cognitive impairment in degenerative parkinsonisms: role of radionuclide brain imaging. $Q \mathrm{~J}$ Nucl Med Mol Imaging. 201256(1):55-67.

2. Emre M, Aarsland D, Brown R, et al. Clinical diagnostic criteria for dementia associated with Parkinson's disease. Mov Disord. 2007;22(12):1689-1707.

3. Aarsland D, Karlsen K. Neuropsychiatric aspects of Parkinson's disease. Curr Psychiatry Rep. 1999;1(1):61-68.

4. Narme P, Tonini A, Khatir F, et al. Non pharmacological treatment for Alzheimer's disease: comparison between musical and non-musical intervention. Geriatr Psychol Neuropsychiatr Vieil. 2012;10(2):215224

5. Holt FE, Birks TPH, Thorgrimsen LM, et al. Aroma therapy for dementia. Cochrane Database Syst Rev. 2003;(3).

6. Fan Z, Aman Y, Ahmed I, et al. Influence of microglial activation on neuronal function in Alzheimer's and Parkinson's disease dementia. Alzheimers Dement. 2014;pii:S1552-5260(14)02501-1 doi:10.1016/j. jalz.2014.06.016.

7. Rolinski M, Fox C, Maidment I, et al. Cholinesterase inhibitors for dementia with Lewy bodies, Parkinson's disease dementia and cognitive impairment in Parkinson's disease. Cochrane Database Syst Rev. 2012;3:CD006504.

8. Drach LM. Drug treatment of dementia with Lewy bodies and Parkinson's disease dementia-common features and differences. Med Monntsschr Pharm. 201134(2):47-52.

9. Dassa A, Amir D. The role of singing familiar songs in encouraging conversation among people with middle to late stage Alzheimer's disease. J Music Ther. 2014;51(2):131-153. 